\title{
Effects of Differences in Working Memory Capacity on Patterns of Word Generation
}

\author{
Mimpei Kawamura \\ Kio University, Nara, Japan; \\ Fukui College of Health Sciences, \\ Fukui, Japan
}

\author{
Yasutaka Kobayashi \\ Fukui General Hospital, \\ Fukui, Japan
}

\author{
Shu Morioka \\ Kio University, \\ Nara, Japan
}

\begin{abstract}
In recent years, it has been reported that WM (working memory) is concerned with word generation, but many points regarding the relationship between the individual differences of WM capacity and the patterns of word generation remain unclear. This study is to investigate these unclear points by using three types of word fluency task with different strategies. Subjects were 28 healthy native speakers of Japanese with an average age of $22.46 \pm$ 3.26 years old. The experimental design included the following two tasks: (1) Japanese reading span test: Subjects were divided into the high-span, middle-span, and low-span WM capacity groups on the basis of the proportion of words recalled correctly; and (2) Word fluency task (category, letter, and verb conditions): In this task, subjects gave as many words as possible that were associated with each stimulus word within $60 \mathrm{~s}$; four stimulus words in each category were used with 12 words in all. As a result, it has become clear that the high-span group with larger WM capacity can generate more words in number than the other groups and is superior in word generating capacity to them and that the low-span group with smaller WM capacity is the highest in the proportion of words generated in the first $15 \mathrm{~s}$ when their vocabulary is abundant, while it is the lowest during $45-60 \mathrm{~s}$ when their vocabulary dries up. The results suggest that WM capacity is involved in the ability to extract appropriate vocabulary from long-term memory information in immediate response.
\end{abstract}

Keywords: word generation, working memory capacity, word fluency task

\section{Introduction}

It is well known that the main regions of the brain associated with language include the left superior/middle temporal gyrus, the inferior parietal lobe, and the left inferior frontal gyrus. The development of technology for making brain activity visible via brain-imaging devices in recent years has also led to numerous reports of more detailed information on the relationship between language and the brain, including functions for which language-related regions are believed to be responsible. Among these, Broca's area, located in the left inferior frontal gyrus, is known to be responsible for language processing, spoken language, and sign language production and understanding (Horwitz et al., 2003). Sakai, Noguchi, Takeuchi, and Watanabe (2002) used fMRI (functional Magnetic Resonance Imaging) to show that Broca's area is also deeply involved in

Mimpei Kawamura, doctorand, Department of Neuro Rehabilitation, Graduate School of Health Science, Kio University; lecturer, Department of Rehabilitation, Speech-Language-Hearing Therapy, Fukui College of Health Sciences.

Yasutaka Kobayashi, Rehabilitation Doctor, Department of Rehabilitation, Fukui General Hospital.

Shu Morioka, Ph.D., Department of Neuro Rehabilitation, Graduate School of Health Science, Kio University. 
grammar processing. The activity of the inferior frontal gyrus, including Broca's area, has also been reported to be significantly affected by differences in the processing loads imposed by tasks themselves (Demb et al., 1995; Chee, Hon, Lee, \& Soon, 2001; Fiebach, Friedrici, Muller, \& von Cramon, 2002).

The activities for which Broca's area is responsible are thus gradually being elucidated by brain-imaging studies. Many points remain unclear with regard to the actual extent of regions with language-related functions, but there is growing awareness of more graded language functions because of their functional connectivity with their surrounding regions. Among these, WM (working memory) is believed to be responsible for the active tasks of extracting contextually appropriate vocabulary, stating one's own opinions, while temporarily storing the other person's words during conversation (Baddeley, 2000). Hulme, Roodenrys, Brown, and Mercer (1995) and Kaneda and Osaka (2007) stated that WM is closely related to linguistic long-term memory information and that WM is involved in the semantic encoding of language, i.e., the task of "imparting meaning to language information”. It is also believed that the dorsolateral prefrontal cortex, an area surrounding the language-related regions described above, is the dominant region corresponding to central executive functioning, which is at the core of WM (Baddeley, 2000). It is, therefore, conjectured that there exists close functional connectivity between WM and language-related regions.

It, therefore, seems that the understanding of the roles of brain function in human language operations is being gradually transformed. Today, word fluency tasks are widely used for the neuropsychological investigation of patterns of word generation. The best-known types of these are category fluency and letter fluency tasks. A category fluency task comprises the production of as many words as possible that belong to a given category within a time limit. This task requires searching accumulated vocabulary and categories of meaning from among formed concepts that are consistent with the instruction and efficiently using semantic memory to generate words that belong to that category. It has been shown that the left mesial temporal lobe is the main center of brain activity during this task (Pihlajamaki et al., 2000). Letter fluency tasks comprise the production of as many words as possible that begin with a particular letter within a time limit. This type of task requires employing the cognitive flexibility to search for words starting with the same letter while suppressing the normal method of using language (i.e., searching for words according to their meanings) (Crawford, Parker, \& McKinlay, 1992). Letter fluency tasks are believed to reflect executive and inhibition functions, which are controlled by the frontal lobe (Lezak, 1995); activity of the left dorsolateral prefrontal cortex has been demonstrated during such tasks (Ravnkidle, Videbech, Rosenberg, Gjedde, \& Gade, 2002; Gaillard et al., 2000). In recent years, more knowledge has also been obtained from verb fluency tasks that require verb generation. Verb fluency tasks require the production of verbs associated with a noun (the stimulus word); many points regarding this process of generation remain unclear. Brain-imaging studies employing verb fluency tasks, however, have shown activity in regions including Broca's area, the left dorsolateral prefrontal cortex, and the anterior cingulate gyrus (Posner \& Raichle, 1994; Peterson, Fox, Posner, Mintun, \& Raichle, 1988, 1989; Horwitz et al., 2003; Shetreet, Palti, Friedmann, \& Hadar, 2007). The strategy used in the process of word generation and the areas of the brain involved therewith thus differ depending on the nature of the stimulus word. Interestingly, the regions of selective brain activity seen in letter fluency and verb fluency tasks overlap with those involved in the central executive functioning characteristic of WM.

Although WM is conjectured to be involved in the process of generating words, no reports have addressed the relationship between WM and word generation. In this study, the authors, therefore, focused on WM capacity and used word fluency tasks demanding different strategies to investigate the effects of individual 
differences in WM capacity on patterns of word generation.

\section{Method}

\section{Subjects}

Subjects were 28 healthy individuals (14 male and 14 female, average age $22.46 \pm 3.26$ years old) who scored $\geq+90 \%$ for right-handedness on the Edinburgh handedness inventory (Oldfield, 1971) and who had no audiovisual medical histories. All subjects were native speakers of Japanese and students at Fukui College of Health Sciences.

\section{Japanese Version of Reading Span Test (M. Osaka \& N. Osaka, 1994; Osaka, 2002)}

This test was administered following the procedure described by M. Osaka and N. Osaka (1994) and Osaka (2002). Scores on the test were calculated, and subjects were divided into three groups showing high (> mean $+1 / 2 S D$ (standard deviation)), middle (within $1 / 2 S D$ of the mean), and low (< mean $-1 / 2 S D$ ) spans on the basis of the proportion of correctly recalled words (Otsuka \& Miyatani, 2007; Fridman \& Miyake, 2005).

\section{Word Fluency Task}

Procedure. The experimental protocol is shown in Figure 1. Category, letter, and verb were set as the three conditions, and four stimulus words were used for each condition. Subjects were asked to say as many words related to the stimulus word as they could speak out loud within a time limit of $60 \mathrm{~s}$. After the $60 \mathrm{~s}$ time limit, a 10 s-rest-period was allowed before the next stimulus word was displayed. Stimulus words were displayed in Japanese on the screen of a personal computer (NEC LaVie LM530/W). In the verb condition, in addition to the above procedure, subjects were asked to express verbs that could be related to the stimulus word, which was a highly familiar noun (e.g., cup $\rightarrow$ drink, hold, wash, etc.).

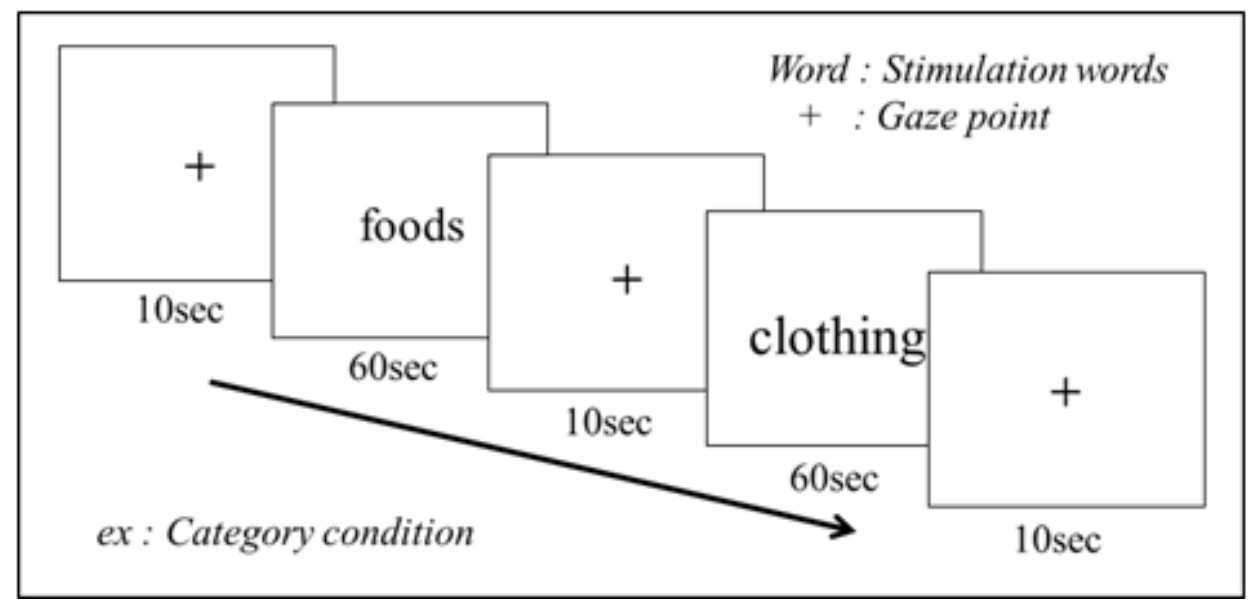

Figure 1. Experimental protocol.

Posture and viewpoint. Subjects were seated in a chair with their heads positioned $60 \mathrm{~cm}$ from the computer screen (see Figure 2). They were instructed to look at either a fixation cross or stimulus word displayed on the computer screen continuously during the tasks. A digital voice recorder (ICD-UX513F, Sony) was used for voice recording of the experimental sessions. 


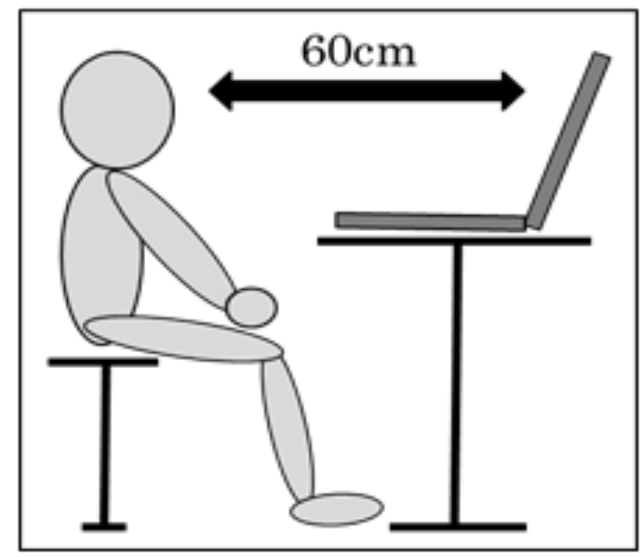

Figure 2. Posture and view point.

Materials. For the category condition, highly familiar words of word familiarity levels 5.001-7.000 from the Nippon Telegraph and Telephone Corporation database series "Lexical Properties of Japanese" (Amano, Kasahara, \& Kondo, 1999) were classified by category, and the four categories containing the largest numbers of words (foods, clothing, occupations, and transportation) were used as stimulus words. For the letter condition, highly familiar words investigated for use as stimulus words in the category condition were classified according to their starting syllables, and the four syllables that started the greatest number of words ( $k a, o, a$, and $k i$ ) were used as stimulus words. For the verb condition, the four most familiar words-one from each of the four categories used in the category condition (udon (wheat noodles), skaato (skirt), pairotto (pilot), and torakku (truck)), were used as stimulus words.

Data analysis. A two-way ANOVA (analysis of variance) was performed with the number of words generated during a trial as the dependent variable and the group and condition factors as independent variables. The $60 \mathrm{~s}$ time limit was then divided into $15 \mathrm{~s}$ intervals, and a two-way ANOVA was performed with the number of words generated during each $15 \mathrm{~s}$ interval as the dependent variable and the condition and time factors as independent variables. A two-way ANOVA was also performed with the proportion of words recalled correctly during each $15 \mathrm{~s}$ interval as the dependent variable and the condition and time factors as independent variables. Multiple comparisons (Tukey-Kramer tests) were performed as post hoc tests.

\section{Results}

\section{Japanese Version of Reading Span Test}

Table 1

Result of Reading Span Test

\begin{tabular}{lrc}
\hline \multirow{2}{*}{ Group } & \multicolumn{2}{c}{ Proportion word (\%) } \\
\cline { 2 - 3 } & $N$ & Mean (SD) \\
\hline High span & 10 & $84.80(4.13)$ \\
Middle span & 9 & $75.79(2.51)$ \\
Low span & 9 & $66.12(6.26)$ \\
\hline
\end{tabular}

Notes. Proportion word stands for the calculated average of correctly recalled-words in all sets after calculating the percentage of correctly recalled-words in each set. Numerical value is the average of each group and $S D$ is the standard deviation.

The average proportion of words recalled correctly for all subjects and $1 / 2$ of the $S D$ are $75.90 \pm 4.48 \%$, 
and when the groups are divided, the high-span group has 10 members, and the middle- and low- span groups have nine members each (see Table 1).

\section{Word Fluency Task}

Table 2 and Figure 3(a) show the word generation average and $S D$. The results of 3 (group) $\times 3$ (condition) two-way ANOVA indicate that significant main effects of both group and condition are evident $\left(F_{(2,75)}=24.23\right.$, $\left.p<0.001 ; F_{(2,75)}=9.09, p<0.001\right)$. Post hoc tests show a significant difference in the number of words generated between the high- and low- span groups in the letter condition $(p<0.05)$. In the verb condition, there are significant differences in the number of words generated between the high-span group and both of the other two groups $(p<0.01)$.

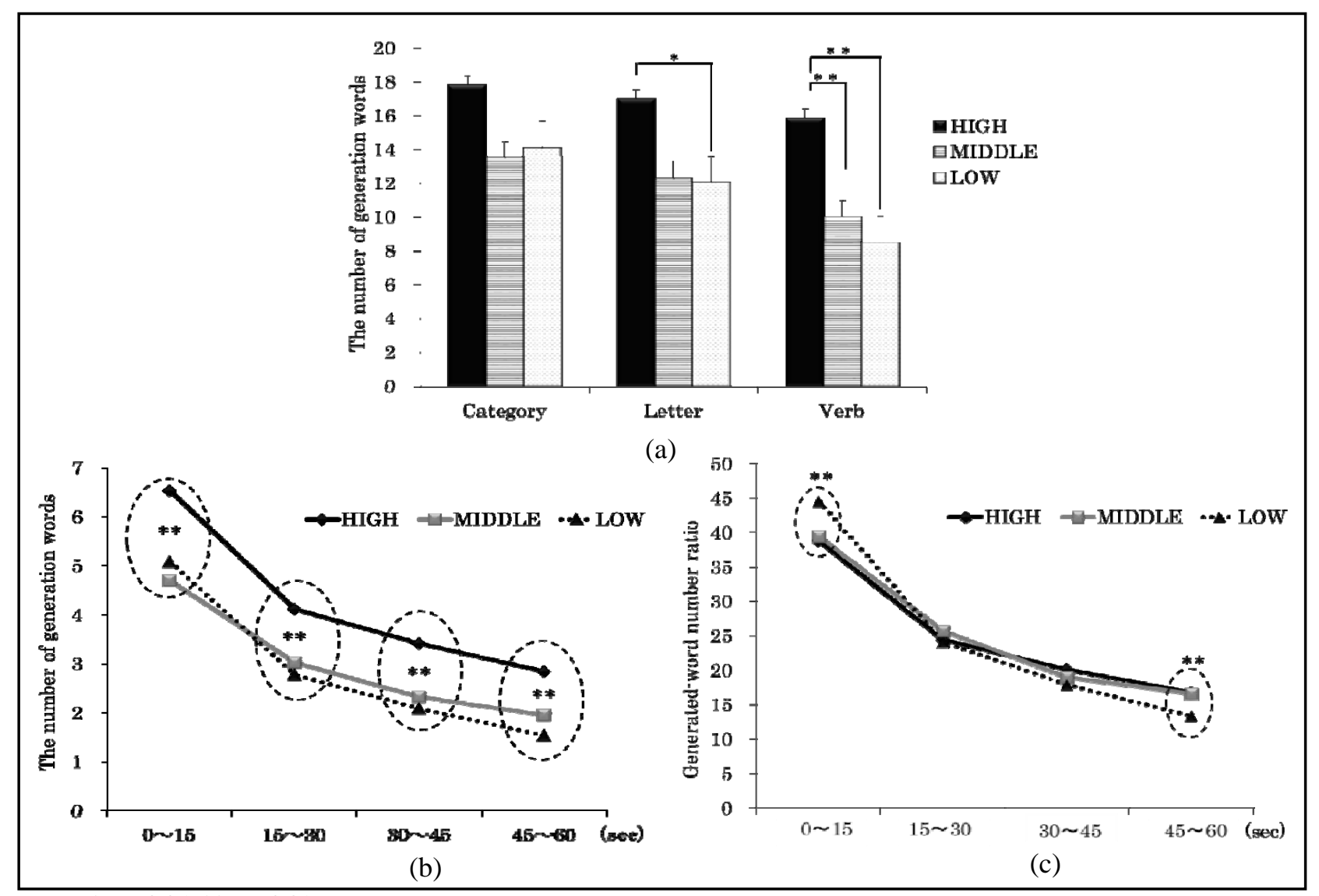

Notes. $* p<0.05,{ }^{* *} p<0.01$.

Figure 3. (a) Average of word-number generated in each condition; (b) Average of word-number generated in each time interval; (c) Average of the proportion of word-number generated in each time interval.

Table 2

Average and SD of Word-Number Generated in Each Different Condition

\begin{tabular}{lllr}
\hline \multirow{2}{*}{ Group } & Category & Letter & Verb \\
\cline { 2 - 4 } & Mean $(S D)$ & Mean $(S D)$ & Mean (SD) \\
\hline High span & $17.83(3.04)$ & $17.03(4.90)$ & $15.85(5.59)$ \\
Middle span & $13.50(4.27)$ & $12.47(3.87)$ & $10.00(3.77)$ \\
Low span & $14.06(3.27)$ & $11.97(3.63)$ & $8.44(3.30)$ \\
\hline
\end{tabular}

Note. Numerical value stands for the average of word-number generated within $60 \mathrm{~s}$ and $S D$ is standard deviation. 
Table 3 and Figure 3(b) show the word generation average and $S D$ of each group during each 15 s time interval. The results of a two-way ANOVA reveal a main effect of group $\left(F_{(3,100)}=34.26, p<0.0000\right)$, and post hoc tests show a significant difference between the number of words generated during each interval by the high-span group and that generated by the other two groups $(p<0.01)$. There is no significant difference between the numbers of words generated during each interval by the middle-span and low-span groups.

Table 3

Average and SD of Word-Number Generated in Each 15 s Time Interval

\begin{tabular}{lllll}
\hline \multirow{2}{*}{ Group } & $0-15 \mathrm{~s}$ & $15-30 \mathrm{~s}$ & $30-45 \mathrm{~s}$ & $45-60 \mathrm{~s}$ \\
\cline { 2 - 5 } & Mean $(S D)$ & Mean $(S D)$ & Mean $(S D)$ & Mean $(S D)$ \\
\hline High span & $6.53(1.15)$ & $4.11(0.81)$ & $3.42(0.88)$ & $2.84(0.68)$ \\
Middle span & $4.69(1.13)$ & $3.02(0.54)$ & $2.32(0.90)$ & $1.95(0.40)$ \\
Low span & $5.08(0.78)$ & $2.78(0.66)$ & $2.09(0.69)$ & $1.54(0.40)$ \\
\hline
\end{tabular}

Note. Numerical value stands for the average of word-number generated in each $15 \mathrm{~s}$ time interval and $S D$ is standard deviation.

Table 4

Average and SD (\%) of the Proportion of Word-Number Generated in Each 15 s Time Interval

\begin{tabular}{lllll}
\hline \multirow{2}{*}{ Group } & $0-15 \mathrm{~s}$ & $15-30 \mathrm{~s}$ & $30-45 \mathrm{~s}$ & $45-60 \mathrm{~s}$ \\
\cline { 2 - 5 } & Mean $(S D)$ & Mean $(S D)$ & Mean $(S D)$ & Mean $(S D)$ \\
\hline High span & $38.81(0.03)$ & $24.39(0.03)$ & $20.02(0.03)$ & $16.78(0.03)$ \\
Middle span & $39.45(0.04)$ & $25.73(0.03)$ & $18.98(0.04)$ & $16.57(0.02)$ \\
Low span & $44.53(0.03)$ & $24.08(0.03)$ & $17.88(0.03)$ & $13.31(0.02)$ \\
\hline
\end{tabular}

Note. Numerical value stands for the average proportion of word-number generated in each $15 \mathrm{~s}$ time interval and $S D$ is standard deviation.

Table 4 and Figure 3(c) show the average and SD of the proportion of the word generation of each group during each $15 \mathrm{~s}$ time interval. The results of a two-way ANOVA indicate a significant main effect of the time factor $\left(F_{(3,75)}=279.0, p<0.0001\right)$, and a significant interaction between the group and time factors $\left(F_{(6,75)}=3.8\right.$, $p<0.01)$. Post hoc tests show that the members of the low-span group correctly recall a significantly higher proportion of their words during the first $15 \mathrm{~s}$ interval $(0-15 \mathrm{~s})$ than the other two groups do $(p<0.01)$, but they recall a significantly smaller proportion of their words during the final interval (45-60 s) than the other two groups do $(p<0.01)$.

\section{Discussion}

In this study, it is found that members of the high-span group who have large WM capacities, have better word formation abilities. This suggests that they may have had fuller access to information from long-term memory or better inhibition function in the process of continuous generation of words compared with the other two groups.

When the $60 \mathrm{~s}$ time limit was divided into $15 \mathrm{~s}$ intervals, although there is no difference in the number of words generated between the middle- and low- span groups, there was a significant difference in the proportions of words correctly recalled by these groups during the $0-15 \mathrm{~s}$ and $45-60 \mathrm{~s}$ intervals. This may be explained in terms of the capacity of constructing effective strategies in real time to express new vocabulary during the 45-60 s time period (when subjects have exhausted their vocabulary expression) rather than in terms of differences in the amount of vocabulary accumulated by members of these three groups. Differences in WM capacity thus seem to be involved in the construction of strategies during the process of word generation. Hurks 
et al. (2010) reported a significant correlation between higher cognitive functioning involved in WM and the deployment of concepts of clustering and switching (Troyer, Moscovitch, \& Winocur, 1997) as patterns of word generation. Differences in WM capacity are thus believed to affect clustering and switching during the process of word generation. In this study, the low-span group with low WM capacity may have therefore been inferior to the other two groups in the cognitive flexibility to perform clustering and switching efficiently in the context of word generation.

Also there are notable differences between groups observed in the verb condition. Verb production first requires that the subject should recognize his/her relationship with an agent or an object. It also requires that the subject should be consciously aware whether or not the selected verb is appropriate to the stimulus word, which means there is a high possibility of requiring WM, which is one of the most important mental faculties in the performance of purposeful actions. The involvement of the left dorsolateral prefrontal cortex and the anterior cingulate gyrus in verb production was mentioned above (Posner \& Raichle, 1994; Peterson et al., 1988, 1989; Horwitz et al., 2003; Shetreet et al., 2007), and interestingly, N. Osaka et al. (2004) reported that the degree of connectivity between the two regions of the left dorsolateral prefrontal cortex and the anterior cingulate gyrus could drive individual differences in WM capacity. Those previous findings indicate a relationship between WM capacity and verb production, and the results of the present study further suggest that differences in WM capacity may affect verb production. It is also surmised, however, that the processing loads imposed by tasks that engage the left inferior frontal gyrus may be a factor in this task (Demb, Desmond, Wanger, Vaidya, Glover, \& Gabrieli, 1995; Chee et al., 2001; Fiebach et al., 2002). Further studies of the connections between verb generation and WM brain functioning are awaited. As verbs are positioned as predicates in Japanese, whether or not WM capacity is involved in predicate operations is also a question for the future.

\section{Conclusions}

In this study, it is shown that individuals with large WM capacity have better word formation ability. The findings suggest that individuals with large WM capacity may have a better ability to extract appropriate vocabulary from information stored in long-term memory. Aspects of executive functioning, such as cognitive flexibility and emergency, may also be important in supporting WM and patterns of word generation. The results also suggest that differences in WM capacity may contribute to verb production. From a clinical perspective, linguistic executive function needs to be kept in mind when developing treatment strategies for the difficulties in recalling words and verbal recall disorders seen in aphasic patients.

\section{References}

Amano, N., Kasahara, K., \& Kondo, T. (1999). NTT Database Series Nihongo-no Goi-Tokusei (Lexical properties of Japanese). Tokyo: Sanseido. (in Japanese)

Baddeley, A. (2000). The episodic buffer: A new component of working memory? Trends in Cognitive Sciences, 4, 417-423.

Chee, M. W., Hon, N., Lee, H. L., \& Soon, C. S. (2001). Relative language proficiency modulates BOLD signal change when bilinguals perform semantic judgments. Neuroimage, 13, 1155-1163.

Crawford, J. R., Parker, D. M., \& McKinlay, W. W. (1992). A handbook of neuropsychological assessment. London: Erlbaum.

Demb, J. B., Desmond, J. E., Wanger, A. D., Vaidya, C. J., Glover, G. H., \& Gabrieli, J. D. (1995). Semantic encoding and retrieval in the left inferior prefrontal cortex: A functional MRI study of task difficulty and process specificity. The Journal of Neuroscience, 15, 5870-5878.

Fiebach, C. J., Friedrici, A. D., Muller, K., \& von Cramon, D. Y. (2002). fMRI evidence for dual routes to the mental lexicon in visual word recognition. Journal of Cognitive Neuroscience, 14, 11-23. 
Fridman, N. P., \& Miyake, A. (2005). Comparison of four scoring methods for the reading span test. Behavior Research Methods, 37, 581-590.

Gaillard, W. D., Herts-Pannier, L., Mott, S. H., Barnett, A. S., LeBihan, D., \& Theodore, W. H. (2000). Functional anatomy of cognitive development: fMRI of verbal fluency in children and adults. Neurology, 54, 180-185.

Horwitz, B., Amunts, K., Bhattacharyya, R., Patkin, D., Jeffries, K., Zilles, K., \& Braun, A. R. (2003). Activation of Broca’s area during the production of spoken and signed language: A combined cytoarchitectonic mapping and PET analysis. Neuropsychologia, 41, 1868-1876.

Hulme, C., Roodenrys, S., Brown, G., \& Mercer, R. (1995). The role of long-term memory machanisms in memory span. British Journal of Psychology, 86, 527-536.

Hurks, P. P., Schrans, D., Meijs, C., Wassenberg, R., Feron, F. J., \& Jolles, J. (2010). Developmental changes in semantic verbal fluency: Analyses of word productivity as a function of time, clustering, and switching. Child Neuropsychology, 16, 366-387.

Kaneda, M., \& Osaka, N. (2007). Role of the executive function in the relationship between verbal working memory and long-term information. The Japanese Journal of Psychology, 78, 235-243.

Lezak, M. D. (1995). Neuropsychological assessment (3rd ed.). New York: Oxford University Press.

Oldfield, R. C. (1971). The assessment and analysis of handedness: The Edinburgh inventory. Neuropsychologia, 9, 97-113.

Osaka, M. (2002). Working memory: The sketchpad in the brain. Tokyo: Shinyosha.

Osaka, M., \& Osaka, N. (1994). Working memory capacity related to reading: Measurement with the Japanese version of reading span test. The Japanese Journal of Psychology, 65, 339-345.

Osaka, N., Osaka, M., Kondo, H., Morishita, M., Fukuyama, H., \& Shibasaki, H. (2004). The neural basis of executive function in working memory: An fMRI study based on individual differences. Neuroimage, 21, 623-631.

Otsuka, K., \& Miyatani, M. (2007). Target words and sentences for Japanese version of the reading span test. Hiroshima Psychological Research, 7, 19-33.

Peterson, S. E., Fox, P. T., Posner, M. I., Mintun, M., \& Raichle, M. E. (1988). Positron emission tomographic studies of the cortical anatomy of signal-word processing. Nature, 331, 585-589.

Peterson, S. E., Fox, P. T., Posner, M. I., Mintun, M., \& Raichle, M. E. (1989). Positron emission tomographic studies of the processing of signal words. Journal of Cognitive Neuroscience, 1, 153-170.

Pihlajamaki, M., Tanila, H., Hanninen, T., Kononen, M., Laakso, M., Partanen, K., \& Aronen, H. J. (2000). Verbal fluency activates the left medial temporal lobe: A functional magnetic resonance imaging study. Annals of Neurology, 47, 470-476.

Posner, M. I., \& Raichle, M. E. (1994). Images of mind. In W. H. Freeman (Ed.). New York: Scientific American Library.

Ravnkidle, B., Videbech, P., Rosenberg, R., Gjedde, A., \& Gade, A. (2002). Putative tests of frontal lobe function: A PET study of brain activation during Stroop's test and verbal fluency. Journal of Experimental Neuropsychology, 24, 534-547.

Sakai, K., Noguchi, Y., Takeuchi, T., \& Watanabe, E. (2002). Selective priming of syntactic processing by event-releted transcranial magnetic stimulation of Broca's area. Neuron, 35, 1177-1182.

Shetreet, E., Palti, D., Friedmann, N., \& Hadar, U. (2007). Cortical representation of verb processing in sentence comprehension: Number of complements, subcategorization, and themetic frames. Cerebral Cortex, 17, 1958-1969.

Troyer, A. K., Moscovitch, M., \& Winocur, G. (1997). Clustering and switching as two components of verbal fluency: Evidence from younger and older healthy individuals. Neuropsychology, 11, 138-146. 